\title{
Therapeutic Management of Conjunctivitis: A Case Report in a Cockatiel and Aseel Bird
}

\author{
Ruchika Lakshmanan $^{1 *}$, K. Senthilkumar ${ }^{2}$ and M. Palanivelrajan ${ }^{3}$ \\ ${ }^{1}$ Department of Wildlife Science, Madras Veterinary College, Chennai-7, India \\ ${ }^{2}$ Post-Graduate Research Institute in Animal Sciences, TANUVAS, \\ Kattupakkam-603 203, India \\ ${ }^{3}$ Department of Wildlife Science, Madras Veterinary College, Chennai-7, India \\ *Corresponding author
}

\section{Keywords \\ Conjunctivitis, \\ Gatifloxacin eye drops, Aseel, \\ Cockatiel, Watery \\ discharge}

Article Info

Accepted:

15 April 2020

Available Online:

10 May 2020

\section{A B S T R A C T}

Conjunctivitis is inflammation of conjunctiva of eye. In recent times, it was found that Gatifloxacin is more safe and effective in treatment of acute form of conjunctivitis than with the same dose of ciprofloxacin. This article presents two case reports in birds wherein Gatifloxacin treatment was used for treatment of conjunctivitis. A female Aseel bird, $1 \frac{1}{2} 2$ years old and a Cockatiel bird, 1 year old was brought to the Avian and Exotic Pet Unit of Madras Veterinary College with the history of swollen eyes and off-feed for the past three days. The birds were unable to open their eyes (right eye for Aseel; left eye for Cockatiel) for the past four days and the unaffected eyes were partially open. On examination of the affected eye, it was noticed that the eye was congested, swollen, crusting over the eyelid and had watery discharge from the eye. The condition was tentatively diagnosed for conjunctivitis. The birds were treated topically with Gatifloxacin eye drops @ 2 drops in the affected eye TA T.I.D for three days. In case of eye discharge, using a clean wash cloth moistened with warm water, gently wiping off the discharge was advised. In addition to this, multivitamin syrup was given to the bird orally. The birds showed marked improvement after five days.

\section{Introduction}

Conjunctivitis is inflammation of the vascular mucous membrane of the eye known as conjunctiva which covers the anterior portion of the eye (bulbar portion), lining the eyelids and the nictitating membrane (palpebral portion) (Mayer and Donnelly, 2012). The conjunctiva is the tissue between the eye of the bird and the eye lid, it is normally not visible, but is light pink in a healthy bird if the eye lid is gently pulled away. In conjunctivitis, the conjunctiva becomes swollen and visible. It is most common eye 
affection occurring in birds. There are numerous etiological factors causing conjunctivitis in birds which include bacterial infections (Chlamydia, mycobacterium, mycoplasma, etc.), fungal (Candida) and viral infections (pox, infectious bronchitis, etc.), trauma, exposure to high doses of ultraviolet light, vitamin-A deficiency, allergy reaction, foreign bodies or irritant and congenital such as microphthalmia in cockatiels (Doneley, 2016). A thorough history must be obtained, including housing conditions, source (wild caught or captive bred), temporal relationship of exposure to other birds, other species ofbirds that may be housed in close proximity to the patient, and whether any other birds in the owner's care similarly have been affected (Abrams et al., 2002). Broad-spectrum topical antibiotic therapy is indicated in cases of conjunctivitis due to infectious cause and it is more frequently accompanied with a concurrent disease (Mayer and Donnelly, 2012). Recently, Gatifloxacin, a fourthgeneration fluoroquinolone, has shown promise with excellent in vitro activity against most pathogens responsible for ocular infections as compared to ciprofloxacin (Mather et al., 2002).

\section{Materials and Methods}

Case 1: A female Aseel bird of $1 \frac{1 / 2}{2}$ years old was brought to the Avian and Exotic Pet Unit of Madras Veterinary College with the history of swollen eyes and off-feed for the past three days. The bird was scratching the eye with the foot which might have even caused injury around the eyes. The bird was unable to open right eye for the past four days. On examination of the right eye, it was noticed that the eye was congested, swollen, crusting over the eyelid and had initial watery discharge which was then turned to be seropurulent from the eye (Figure 1). Eye swabs for bacterial culture were collected. No other clinical signs of concurrent illness were noticed. Hence, the chicken was treated topically with Gatifloxacin eye drops @ 2 drops in the affected eye TA T.I.D for three days. In case of eye discharge, using a clean wash cloth moistened with warm water, gently wiping off the discharge was advised. In addition to this, multivitamin syrup was given to the bird orally@ 2drops orally given every morning everyday.

Case 2: Similar to above mentioned case, a Cockatiel bird of 1 year old was brought to the Avian and Exotic Pet Unit of Madras Veterinary College with the history of swollen eyes and was anorexic for the past three days. The bird was continuously raking the eye against the enclosure and scratching with the foot. The bird was unable to open left eye for the past four days and the right eye was partially open (Figure 2 and 3). On examination of the eyes, it was noticed that the eyes were congested, swollen and had watery to mild seropurulent discharge from the eye. Further clinical signs even included excessive preening and rubbing of eyes with no evidence of concurrent systemic illness. Similarly, as the above case swabs from the ocular discharge were collected for bacterial culture. The bird was treated topically with Gatifloxacin eye drops @ 2 drops in the affected eye TA T.I.D for three days. In case of eye discharge, using a clean wash cloth moistened with warm water, gently wiping off the discharge was advised. In addition to this, multivitamin syrup was given to the bird orally@2drops orally given every morning everyday.

\section{Results and Discussion}

In both cases bacterial culture report showed presence of gram positive bacteria and sensitive to broad spectrum antibiotics. The above mentioned treatment showed marked improvement in both the birds after 5 days (Figure 4). The Gatifloxacin eye drops (Higati 
eye drops) showed complete healing and recovery in five days. The birds were able to open the eyes and found difficult to view objects. As there was presence of no systemic illness, parental antibiotic therapy was not used and acute conjunctivitis was treated with topical application of the Gatifloxacin eye drops.

The present clinical findings were in accordance with Abrams et al., (2002). As discussed by Abrams et al., (2002) and Mayer and Donnelly (2012), topical broad spectrum antibiotic therapy is proved to be the best for treating primary conjunctivitis. Mather et al., 2002 cited that Gatifloxacin, which is the fourth generation fluoroquinolones, is a new weapon in the arsenal of ophthalmic antibiotics. Though Parmar et al., 2006 highlighted that Gatifloxacin showed complete healing and recovery in case of bacterial keratitis as compared to that of ciprofloxacin even though the doses were same of two drugs but the mean time of healing was found to be the same for both drugs. Similarly, Jensen et al., (2006), even quoted that Gatifloxacin is safe and effective in treatment of acute ophthalmic affections.

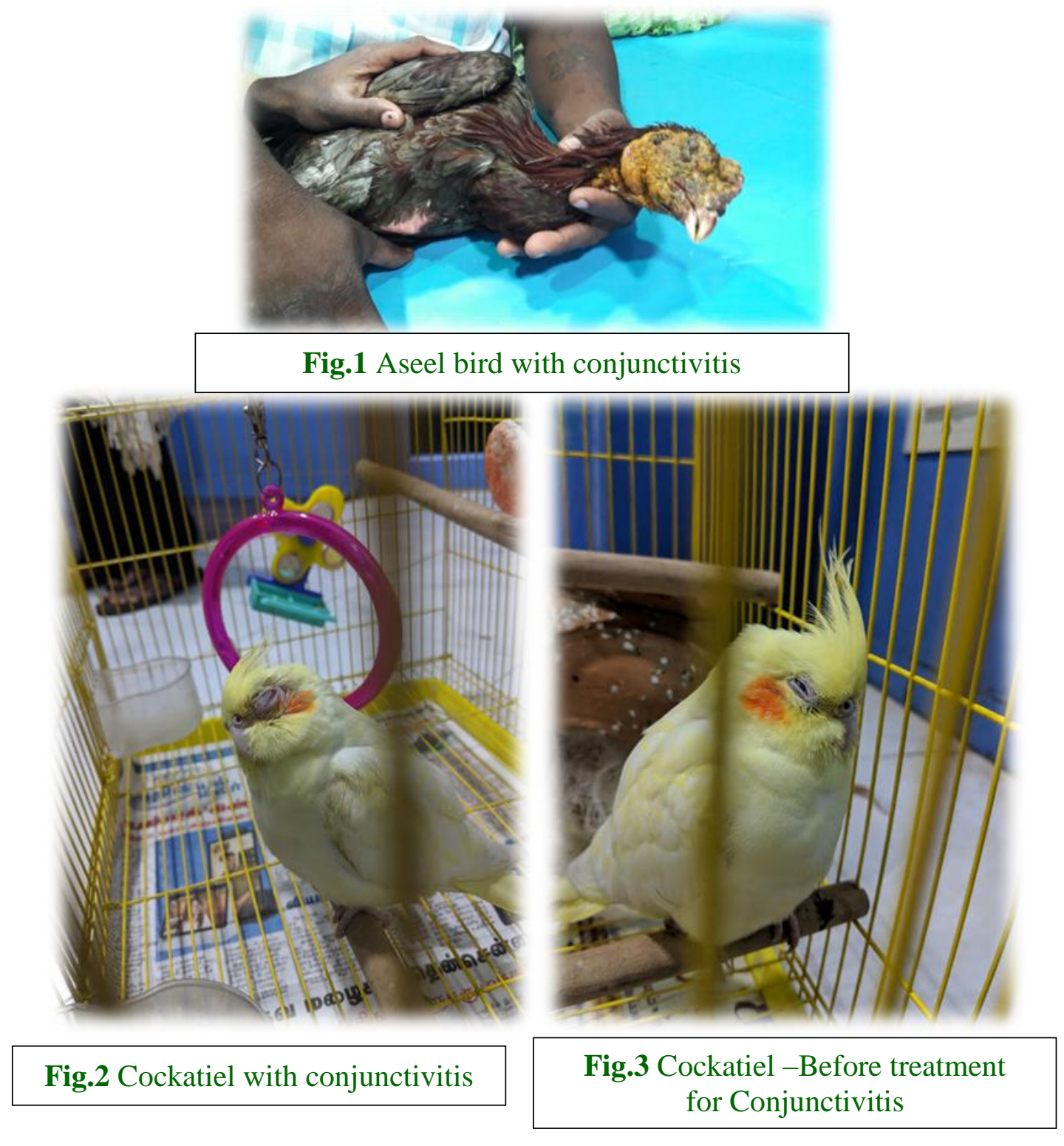




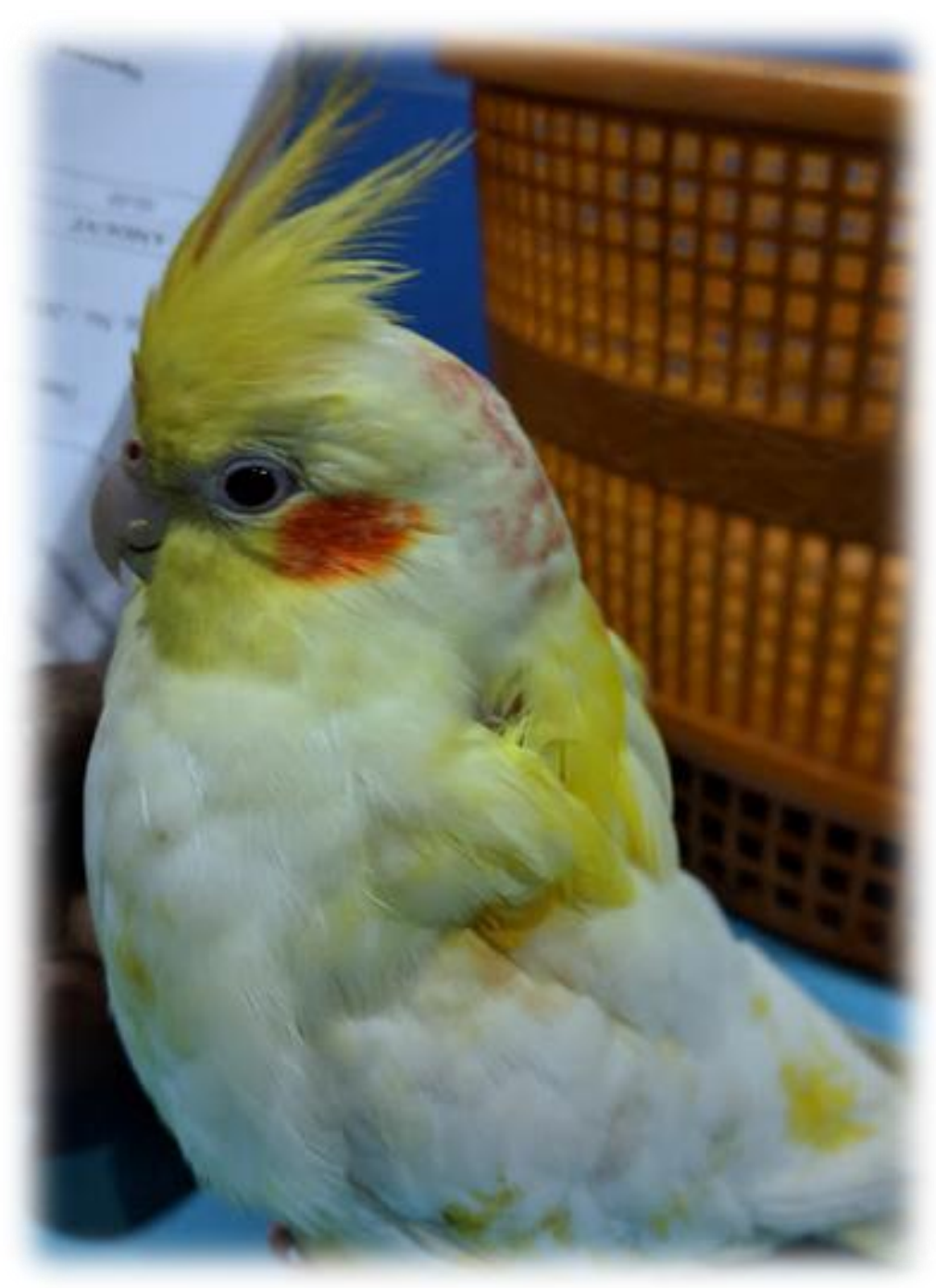

Fig.4 Cockatiel - post treatment for conjunctivitis

\section{Acknowledgement}

The authors are highly grateful to, the Dean, Faculty of Basic Sciences, TANUVAS and the Dean, Madras Veterinary College for permission and all possible support. The authors are also thankful to Dr. C. Sreekumar, Ph.D., Head of Department, Department of Wildlife Science, Madras Veterinary College, TANUVAS for valuable suggestion in this study.

\section{References}

Abrams, G.A., Paul-Murphy, J. and Murphy, C.J., 2002. Conjunctivitis in birds. Veterinary Clinics: Exotic Animal Practice, 5(2), pp.287-309.

Doneley, B., 2016. Disorders of Eye. Avian medicine and surgery in practice: companion and aviary birds. CRC press.

Jensen, H.G., Prajna, V., Vajpayee, R., Trocme, S., Davitt III, W.F., Liu, R. 
and Safyan, E., 2006. Effect of Gatifloxacin $0.3 \%$ and Ciprofloxacin $0.3 \%$ on Acute Bacterial Corneal Ulcer Isolates. Investigative Ophthalmology \& Visual Science, 47(13), pp.1923-1923.

Mather, R., Karenchak, L.M., Romanowski, E.G. and Kowalski, R.P., 2002. Fourth generation fluoroquinolones: new weapons in the arsenal of ophthalmic antibiotics. American journal of ophthalmology, 133(4), pp.463-466.

Mayer, J. and Donnelly, T.M., 2012. Clinical veterinary advisor: birds and exotic pets. Elsevier Health Sciences.
Parmar, P., Salman, A., Kalavathy, C.M., Kaliamurthy, J., Prasanth, D.A., Thomas, P.A. and Jesudasan, C.A.N., 2006. Comparison of topical gatifloxacin $0.3 \%$ and ciprofloxacin $0.3 \%$ for the treatment of bacterial keratitis. American journal of ophthalmology, 141(2), pp.282-286.

Ritchie, B.W., Harrison, G.J. and Harrison, L.R., $\quad$ 1994. Chapter: 26 Ophthalmology. Avian medicine: principles and application. HBD International, Incorporated.

\section{How to cite this article:}

Ruchika Lakshmanan, K. Senthilkumar and Palanivelrajan, M. 2020. Therapeutic Management of Conjunctivitis: A Case Report in a Cockatiel and Aseel Bird. Int.J.Curr.Microbiol.App.Sci. 9(05): 2063-2067. doi: https://doi.org/10.20546/ijcmas.2020.905.236 